\title{
Uma análise em livros didáticos de matemática dos anos finais do ensino fundamental acerca da proposta do ensino de polígonos sob a ótica da teoria de Van Hiele.
}

\author{
An analysis in textbooks of mathematics of the final years of elementary school about \\ the proposal of teaching polygons from the Van Hiele theory.
}

Franklin Fernando Ferreira Pachêco pacheco.franklin9@gmail.com

Gisele Ferreira Pachêco gisele.ferreira4@gmail.com

Anderson Douglas Pereira Rodrigues da Silva anderdouglasprs@gmail.com

\begin{abstract}
Resumo
A pesquisa teve como objetivo central analisar a proposta do ensino de polígonos na coleção dos livros didáticos mais utilizados no município de São Vicente Férrer do Estado de Pernambuco, tendo como aporte teórico o modelo proposto por van Hiele. Para alcançarmos o objetivo da pesquisa foi necessário identificarmos inicialmente, junto à Secretaria de Educação do município, os livros didáticos mais utilizados no II ciclo do Ensino Fundamental. A realização da análise desses livros foi fundamental na identificação das relações existentes entre os autores desses livros e o modelo teórico adotado por Van Hiele. Levamos, ainda, em consideração as recomendações dos PCNs de Matemática para esses ciclos de escolaridade. A escolha desse recurso didático foi por considerar um instrumento relevante e de referência para os professores, sobretudo, nas atividades realizadas em sala de aula. Nossa fundamentação teórica esteve apoiada no modelo proposto por Van Hiele que adota níveis de 1 a 5 de desenvolvimento do pensamento geométrico que podem ser utilizados na organização de vários conteúdos de geometria e em diversos níveis de escolaridade. Trata-se, portanto, de uma pesquisa qualitativa baseada na análise de conteúdo de geometria, em especial, os polígonos. A partir das análises observamos que o autor, de modo geral, propõe o estudo dos polígonos de forma sequenciada, ou seja, define, exemplifica e só então, apresenta as atividades.
\end{abstract}

Palavras Chaves: Polígonos; Livros didáticos; Desenvolvimento do pensamento geométrico.

\begin{abstract}
The research was mainly aimed to analyze the proposal of polygons teaching in the collection of textbooks commonly used in São Vicente Férrer municipality of Pernambuco, having as theoretical support the model proposed by van Hiele. To achieve the objective of the research was necessary identify initially by the Municipal Department of Education, textbooks commonly used in primary education II cycle. The completion of the analysis of these books was instrumental in identifying the relationship between the authors of these books and the theoretical model adopted by Van Hiele. We take also into account the recommendations of Mathematics NCPs for these education cycles. The choice of this teaching resource was considering a relevant tool and reference for teachers, especially in the activities developed in the classroom. Our theoretical foundation was supported by the model proposed by Van Hiele Adopting levels 1-5 of development of geometric thinking that can be used in the organization of various geometry content and at various levels of education. It is therefore a qualitative study based on the geometry of content analysis, especially polygons. From the analysis we found that the author, in general, proposes the study of polygons sequenced manner, ie, sets, exemplifies and only then, presents the activities.
\end{abstract}

Keywords: Development of geometric thinking; Textbook; Polygons. 


\section{INTRODUÇÃO}

A geometria surgiu na antiguidade a partir da necessidade humana que sentiram a necessidade de medir, dividir terras, construir casas, calcular distâncias e dentre outros. Seus primeiros indícios aconteceram na agricultura, em civilizações como as dos Egípcios, Babilônicos e etc.

Quando o homem adquiriu percepção de conhecimentos em relação a geometria, como, noções de distâncias, retas e figuras geométricas, técnicas foram elaboradas servindo de base para uma melhor adaptação do homem ao seu meio, sendo posteriormente aperfeiçoadas com seus conhecimentos prévios já existentes. Concordamos com Eves (1994 Apud Maziero,2015, p.15) ao enfatizar que inúmeras circunstâncias da vida, até mesmo do homem mais primitivo levavam o certo montante de descobertas geométricas subconscientes. A noção de distância foi, sem dúvidas, um dos primeiros conceitos geométricos a ser desenvolvido. A necessidade de delimitar a terra levou a noção de figuras geométricas simples, tais como retângulos, quadrados e triângulos. Outros conceitos geométricos simples, como as noções de vertical, paralela e perpendicular, teriam sido sugeridos por construções de muros e moradias.

Gradativamente esta área de conhecimento foi sendo aperfeiçoada e como produto disto conhecemos a geometria cotidiana que se apresenta nos livros didáticos. Quanto a isto, Araújo (2007, p.1) ressalta que "[...] primeiro, ela surgiu como uma geometria intuitiva, passando em "seguida" a uma geometria cientifica e desta, para o que temos na atualidade, que é entre tantas outras uma geometria demonstrativa".

O percussor da Geometria Demonstrativa a qual conhecemos na cotidianamente foi Tales de Mileto. Posteriormente ela foi aperfeiçoada e transcrita para que estes conhecimentos chegassem ao âmbito escolar.

Atualmente a geometria é um ramo da matemática e este conhecimento faz parte das propostas curriculares da educação básica. Percebe- se que os conteúdos relacionados a este geralmente são apresentadas nos capítulos finais dos livros didáticos de matemática. Observase, entretanto, que a geometria, apesar de ser um conhecimento necessário, pois ela permite enxergar, como, por exemplos as formas geométricas nas variadas situações do nosso cotidiano, seja em prédios, na natureza e etc, há um grande descaso do seu ensino nas escolas. Pesquisas na área como, por exemplo, a de Peres (1991), aponta para um certo descaso no 
ensino da geometria, ao enfatizar que "a geometria está ausente ou quase ausente de sala de aula".

Apesar desta omissão de seu ensino, é perceptível a vasta aplicação da geometria na vida prática, pois afinal ela integraliza os blocos de conteúdos de Grandezas e Medidas, assim como, Espaço e Forma, que apresentam relações diretas com as formas geométricas e essas relações são relevantes para resolver situações do dia a dia. Portanto, é um conhecimento necessário para o desenvolvimento individual e para a compreensão de mundo e, por isso mesmo, faz parte de todos os currículos escolares da educação básica. Nesse contexto, Lorenzatto (1995) diz que o estudo da geometria proporciona ao indivíduo, experimentar, descobrir, fazer conjecturas, enfim, valoriza o processo da construção de conhecimentos e que aquele que não conhece a geometria terá uma leitura interpretativa do mundo incompleta.

Nesta perspectiva, o presente trabalho aborda a seguinte problemática, que relação pode ser estabelecida entre o modelo de desenvolvimento do pensamento geométrico proposto pelos Van Hiele e a proposta de ensino de polígonos nos livros didáticos dos anos finais do ensino fundamental mais utilizados no município de São Vicente Férrer / PE?

É nesse contexto que se insere a nossa pesquisa que teve como objetivo geral, analisar a proposta de ensino de polígonos na coleção de livros didáticos mais utilizados no município de São Vicente Férrer / PE, segundo o modelo de Van Hiele. E, como objetivos específicos, fazer um levantamento, junto a secretaria de educação do município, da coleção de livros didáticos mais usados no ensino fundamental da rede pública do município; buscando, identificar nos livros didáticos o (s) tópico (s) referente ao conteúdo de polígono selecionado neste estudo, com o propósito de confrontar as atividades propostas nessa coleção com as características dos níveis de desenvolvimento do pensamento geométrico dos Van Hiele.

Embasamos nossa pesquisa no Modelo de desenvolvimento do pensamento geométrico proposto por Van Hiele, que tem o papel de avaliar as habilidades dos alunos sobre os conteúdos em geometria, servindo como um guia para o professor compreender em que nível seus alunos se encontram em relação ao conteúdo. Sobre isto, Rodrigues (2014, p.1), enfatiza "O modelo de Van Hiele de desenvolvimento do pensamento geométrico tem sido utilizado para facilitar a compreensão de conteúdos em geometria, enriquecendo o espaço de ensino e aprendizagem". Portanto, neste artigo faremos uma breve explanação sobre a fundamentação teórica, metodologia e apresentaremos a análise de dados. 


\section{FUNDAMENTAÇÃO TEÓRICA}

\subsection{Os conhecimentos Geométricos e o Modelo de Van Hiele}

Desde o surgimento dos conhecimentos geométricos, na antiguidade, por meio das necessidades dos homens, percebemos que com sua evolução no decorrer dos anos estes atualmente exercem grande relevância para a sociedade. Nas diversas situações do nosso cotidiano, notamos que esta área da Matemática, encontra- se presente em nossas vidas, como, por exemplo, nas construções de prédios, objetos eletrônicos, e dente outros. Sendo assim, concordamos com Guerato $(2008$, p.13) ao mencionar que "observando a natureza e os diversos objetos criados pelo homem, notamos que a geometria está em todos os lugares".

Sabendo- se que a geometria está presente em nossa trajetória de vida, enfatizamos que ela é essencial para o currículo, pois estes conhecimentos permitem aos alunos desenvolver o pensamento geométrico capaz de perceber que a geometria está presente em todos os lugares. Conforme, Brasil (1998, p.122), ressalta "a geometria (...) desempenha um papel fundamental no currículo, na medida em que possibilita ao aluno desenvolver um tipo de pensamento particular para compreender, descrever e representar de forma organizada, o mundo em que vive".

Acerca desta questão, concordamos com Lorenzato (1995) ao enfatizar que sem estudar geometria as pessoas não desenvolvem o pensar geométrico e, sem essa habilidade elas, dificilmente conseguirão resolver as situações de vida que forem geometrizadas.

Mesmo sabendo- se que a geometria é relevante para a formação dos alunos, nota- se sua ausência mediante seu ensino, e, percebemos como, Pavanello (1993 apud Guimarães, 2006, p.6), enfatiza "o abandono da geometria tem sido um fato marcante na educação mundial".

Diante deste quadro, a respeito da ausência do ensino da geometria utilizamos o Modelo de Van Hiele, para nossa investigação. Criado pelo casal Dina Van Hiele e Pierre Mario Van Hiele, dois educadores holandeses propuseram em seu trabalho acadêmico de doutorado na Universidade de Utrecht, uma teoria sobre o aprendizado em geometria, com o intuito de verificar a maturidade dos alunos acerca dos conhecimentos geométricos, tendo, por base suas dificuldades.

O modelo de Van Hiele concebe cinco níveis de forma hierárquica em que o aluno só atinge um determinado nível após passar pelo anterior e consequentemente passa atingir o 
raciocínio geométrico depois de passar por todos os níveis. Sobre isto, Nasser (1997 apud VIEIRA, 2010, p.17-18), enfatiza "o modelo sugere que os alunos progridem segundo uma sequência (sic) de níveis de compreensão de conceitos, enquanto eles aprendem geometria”.

O progresso de um nível para outro, é baseado através de vivencias realizadas por meio de atividades, ou seja, esta "passagem" dependem mais da aprendizagem dos alunos do que a sua maturação e/ou idade. Nesta perspectiva, Alves e Sampaio (2010, p.70), mencionam que "[...] numa sala de aula, cada aluno pensa em diferentes níveis e, além disso, eles apresentam modos de pensar diferentes dos professores, pois costumam utilizar com frequência palavras e objetos distintos dos empregados pelos professores e livros”.

No nosso caso, utilizaremos a Teoria de Van Hiele para detectar nas atividades da coleção de livro didáticos proposto por Edwaldo Bianchini (2006), qual nível de conhecimentos geométricos os alunos devem se enquadrar para resolve-las.

A seguir, apresentaremos o quadro abaixo apresentado por Alves e Sampaio (2010, P.70), que resume os níveis de compreensão proposto por van Hiele.

Quadro 1: Níveis de compreensão do modelo de Van Hiele

\begin{tabular}{|c|c|}
\hline Níveis de Compreensão & Características \\
\hline $\begin{array}{c}\text { Visualização ou reconhecimento } \\
\text { (Nível 1) }\end{array}$ & $\begin{array}{c}\text { - Reconhece visualmente uma figura } \\
\text { geométrica; } \\
\text { - Tem condições de aprender o vocabulário } \\
\text { geométrico; } \\
\text { - Não reconhece ainda as propriedades de } \\
\text { identificação de uma determinada figura. }\end{array}$ \\
\hline $\begin{array}{l}\text { Análise } \\
\text { (Nível 2) }\end{array}$ & $\begin{array}{l}\text { - Identifica as propriedades de uma } \\
\text { determinada figura; } \\
\text { - Não faz inclusão de classes. }\end{array}$ \\
\hline $\begin{array}{c}\text { Dedução informal ou Ordenação } \\
\text { (Nível 3) }\end{array}$ & $\begin{array}{l}\text { - Já é capaz de fazer inclusão de classes; } \\
\text { - Acompanha uma prova formal, mas não é } \\
\text { capaz de construir outra. }\end{array}$ \\
\hline $\begin{array}{l}\text { Dedução Formal } \\
\text { (Nível 4) }\end{array}$ & $\begin{array}{c}\text { - É capaz de fazer provas formais; } \\
\text { - Raciocina num contexto de um sistema } \\
\text { matemático completo. }\end{array}$ \\
\hline $\begin{array}{l}\text { Rigor } \\
\text { (Nível 5) }\end{array}$ & $\begin{array}{l}\text { - É capaz de comparar sistemas baseados em } \\
\text { diferentes axiomas; } \\
\text { - É neste nível que as geometrias não- } \\
\text { euclidianas são compreendidas. }\end{array}$ \\
\hline
\end{tabular}

Fonte: Alves e Sampaio (2010, p.70) 
Utilizaremos os cinco níveis do Modelo de Van Hiele, para confrontar as atividades apresentadas na coleção de livros de Edwaldo Bianchini (2006).

O nível da visualização, é demarcado pelo reconhecimento das figuras pelas suas aparências, logo, os alunos pertencentes a esse nível conseguem conhecer ou reproduzir figuras pela sua forma e não pelas suas propriedades.

O nível da análise, é determinado pela percepção de perceber as características das figuras e conseguir descrever suas propriedades.

No nível da dedução informal, a dedução é o fator predominante, pois é aqui que os alunos usam seu raciocínio para pensar sobre determinadas figuras apenas por meio de suas propriedades.

No nível, caracterizado como dedução formal, as construções de definições baseiam-se na percepção do necessário e do suficiente, isto é conforme Silva (2011, p. 23) "O nível da dedução, nele o objetivo é compreender o significado da dedução das teorias geométricas de uma forma mais complexa, dentro de um sistema axiomático”.

O último nível, e o mais elevado do modelo de Van Hiele é denominado rigor, e, é alcançado por poucos alunos, pois, diz respeito aos aspectos abstratos formais de dedução. $\mathrm{Ou}$ seja, ele é caracterizado por construção de hipóteses num sistema axiomático, além dos axiomas Euclidiano.

\section{METODOLOGIA DA PESQUISA}

A pesquisa é classificada como documental de cunho qualitativa, visto que, com base na problemática, o objetivo central da pesquisa foi o de analisar a proposta do ensino de polígonos na coleção de livros didáticos mais utilizados no Município de São Vicente Férrer/ PE, tendo como referência a teoria de Van Hiele.

Optamos por seguir uma linha de pesquisa de cunho de abordagem metodológica qualitativa, pelo fato da mesma buscar em sua especificidade o cunho qualitativo de abordagem e percepção de resultados. Pois, este tipo de pesquisa se opõe a pesquisa quantitativa pois considera que seus resultados não estão apenas relacionados a "números", isto é, esta não busca apenas o quantitativo. Neste contexto, concordamos com Chizzotti (2003), que "a pesquisa qualitativa recobre, hoje, um campo transdisciplinar, envolvendo as ciências humanas e sociais, assumindo tradições ou multiparadigmas de análise, derivadas do 
positivismo, da teoria crítica e do construtivismo adotando multimétodos de investigação para o estudo [...]".

No que diz respeito a pesquisa documental, concordamos com Gil (2008), ao enfatizar que se assemelha muito à pesquisa bibliográfica. E, que a distinção entre ambas está na natureza das fontes, ou seja, enquanto a pesquisa bibliográfica se utiliza fundamentalmente das contribuições dos diversos autores sobre determinado assunto, as pesquisas documentais podem ser reelaboradas de acordo com os objetivos da pesquisa.

Diante dos parágrafos expostos, esta pesquisa busca analisar a relação estabelecida entre o desenvolvimento do pensamento geométrico de Van Hiele e a proposta de ensino de polígonos, apresentadas na coleção de livros didáticos dos anos finais do ensino fundamental.

Nosso instrumento de coleta de dados, foi a coleção de livros didáticos intitulada: "Matemática" obra composta por em quatro volumes, referente aos anos finais do ensino fundamental ( $6^{\circ}$ ao $9^{\circ}$ ano). Tendo, como autor Edwaldo Bianchini, sendo a $6^{\mathrm{a}}$ edição das obras, na editora Moderna- São Paulo 2006. A escolha desta coleção de livros didáticos foi devido as escolas da rede Municipal localizadas no município de (São Vicente Férrer /PE) a adotarem como o mais utilizado durante o ano de 2013.

Nesta perspectiva, a respeito da fonte de coleta de dados de uma pesquisa documental Gil (2008, p. 147), menciona:

\footnotetext{
Essas fontes documentais são capazes de proporcionar ao pesquisador dados em quantidade e qualidade suficiente para evitar a perda de tempo e o constrangimento que caracterizam muitas das pesquisas em que os dados são obtidos diretamente das pessoas. Sem contar que em muitos casos só se torna possível realizar uma investigação social por meio de documentos.
}

De acordo com o exposto, ressaltamos que não há nenhum empecilho na análise, pois o estudo do conteúdo de polígonos propostas em coleções de livros didáticos abordam propostas diferentes de ensino - aprendizagem, e, por isso aderimos aos níveis de compreensão do Modelo de Van Hiele, por se tratar de uma teoria ao qual relaciona- se ao ensino - aprendizagem em geometria. E, além disso, pelo fato dos PCN's apresentarem durante todo o decorrer do Ensino Fundamental o estudo dos polígonos, com atividades exploratórias que relacionam com os níveis de compreensão do modelo de Van Hiele, como, 
por exemplo, noções de figuras geométricas, resoluções de situações - problemas, semelhanças de figuras e et al.

Para identificar na coleção os livros didáticos, tomamos as seguintes nomenclaturas: L1, L2, L3 e L4, estas nomenclaturas correspondem respectivamente, ao $6^{\circ}, 7^{\circ}, 8^{\circ}$ e $9^{\circ}$ ano do Ensino Fundamental.

Procurando responder a problemática, como também alcançar nossos objetivos descritos no início da pesquisa, nossos dados obtidos foram provenientes por meio das atividades apresentadas sobre polígonos pelo autor da coleção dos livros didáticos mais utilizados no município de São Vicente Férrer /PE, sendo confrontada de forma qualitativa pelos níveis de compreensão do Modelo de Van Hiele.

Ainda, sobre a análise de dados concordamos com Goldenberg (2007, p.53) ao enfatizar que "os dados qualitativos consistem em descrições detalhadas de situações com o objeto de compreender os indivíduos em seus próprios termos. Estes dados não são padronizáveis como os dados quantitativos, obrigando o pesquisador a ter flexibilidade e criatividade no momento de coletá-los e analisá-los".

Portanto, nos livros didáticos, foram observadas as atividades de polígonos propostas pelo autor da obra, identificando se este estudo estabelece relações entre o modelo de desenvolvimento do pensamento geométrico de Van Hiele.

\section{ANÁLISE DE DADOS}

Foram analisadas, na coleção de livros didáticos, atividades que variam do nível da Visualização à Dedução formal, pois, o nível caracterizado como rigor não foi apresentado segundo a proposta de ensino de polígonos pelo autor da coleção. Concordamos com Silva (2011, p. 34) pois, para ele, "Em razão da complexidade envolvida nos dois últimos níveis da teoria de Van Hiele, compreendemos que a proposta para esse nível de escolaridade só atingirá adequadamente no máximo até o nível 4 do Modelo”.

A seguir, apresentaremos os tópicos dos conteúdos sobre polígonos abordados nos livros didáticos nos anos finais do ensino fundamental - coleção Matemática (Edwaldo Bianchini) 
Quadro 2: Tópicos referentes ao conteúdo de polígonos abordado na coleção de livros didáticos

\begin{tabular}{|c|c|}
\hline $\begin{array}{l}\text { Ano a ser } \\
\text { trabalhado }\end{array}$ & Conteúdos \\
\hline \multirow{3}{*}{$6^{\circ}$ ano } & $\begin{array}{l}\text { Polígonos: Linhas poligonais; Interior, exterior e convexidade; Polígonos; } \\
\text { Elementos de um polígono e Classificação dos polígonos. }\end{array}$ \\
\hline & $\begin{array}{l}\text { Triângulos: Classificação quantos aos lados e ângulos; construção de } \\
\text { triângulos. }\end{array}$ \\
\hline & Quadriláteros e suas classificações. \\
\hline $7^{\circ}$ ano & $\begin{array}{l}\text { Simetria: Reconhecendo a simetria; figuras com mais um eixo de simetria; } \\
\text { polígonos regulares; simetria em relação a uma reta. }\end{array}$ \\
\hline \multirow{3}{*}{$8^{\circ}$ ano } & $\begin{array}{l}\text { Estudo dos polígonos: Os polígonos; número de diagonais de um polígono; } \\
\text { soma das medidas dos ângulos internos de um polígono; soma das medidas } \\
\text { dos ângulos internos de um triângulo; soma das medidas dos ângulos } \\
\text { internos de um polígono de n lados; soma das medidas dos ângulos } \\
\text { externos de um polígono; soma das medidas dos ângulos externos de um } \\
\text { polígono de n lados; polígonos regulares; congruência de polígonos; } \\
\text { elementos correspondentes em polígonos congruentes; transformações } \\
\text { geométricas que geram figuras congruentes; reflexão; translação; rotação. }\end{array}$ \\
\hline & $\begin{array}{l}\text { Estudos dos triângulos: Principais elementos de um triângulo; classificação } \\
\text { e construção de triângulos; outros elementos de uns triângulos; mediana; } \\
\text { bissetriz; altura; congruência de triângulos; casos de congruência de } \\
\text { triângulos; demonstrações Geométricas; noções primitivas e postulados; } \\
\text { teoremas; a congruência de triângulos nas demonstrações geométricas; } \\
\text { propriedade de um triângulo isósceles; outras propriedades. }\end{array}$ \\
\hline & $\begin{array}{l}\text { Estudo dos quadriláteros: Quadriláteros; elementos dos quadriláteros; } \\
\text { ângulos de um quadriláteros; paralelogramos; propriedades dos } \\
\text { paralelogramos; propriedades dos retângulos; propriedades dos losangos; } \\
\text { propriedades dos quadrados. }\end{array}$ \\
\hline $9^{\circ}$ ano & $\begin{array}{l}\text { Polígonos regulares: Polígonos regulares; elementos dos polígonos } \\
\text { regulares; relações métricas nos polígonos regulares; quadrado inscrito; } \\
\text { hexágono inscrito; triângulo equilátero inscrito; área do polígono regular; } \\
\text { área de um círculo. }\end{array}$ \\
\hline
\end{tabular}

Fonte: Coleção de Edwaldo Bianchini (2006). 
Diante dos tópicos dos conteúdos sobre os polígonos que são apresentados na coleção de livros didáticos, a seguir, apresentaremos, algumas das atividades que o autor apresenta nos mesmos.

As atividades, a seguir, expostas foram confrontadas mediante os níveis de compreensão do modelo de Van Hiele, sendo assim, em cada livro analisado por sua vez serão expostas as atividades que requerem maiores conhecimentos geométricos para resolvé- las, pois como as os níveis de compreensão do Modelo de Van Hiele, é construído de maneira hierárquica, consequentemente será necessário conhecimento prévio dos níveis anteriores. Por exemplo, se for exposto uma atividade que se enquadra ao nível da análise, logicamente será necessário conceber conhecimentos geométricos do nível anterior, neste caso o da visualização, e assim sucessivamente.

No L1, livro didático do $6^{\circ}$ ano, observamos, mediante nossa análise que as atividades se enquadram nos níveis da visualização e análise. Por isso, apresentaremos uma atividade referete a triângulos, classificada no nível da análise.

Figura 01: Triângulos



Fonte: Bianchini(2006, p.262)

Assim, como no livro do $6^{\circ}$ ano, o L2, apresenta atividades que se enquadram nos níveis da visualização e análise. Por este motivo, a atividade a seguir, apresentada se enquadra no nível da análise, conforme vemos na figura 02.

Figura 02: Polígonos

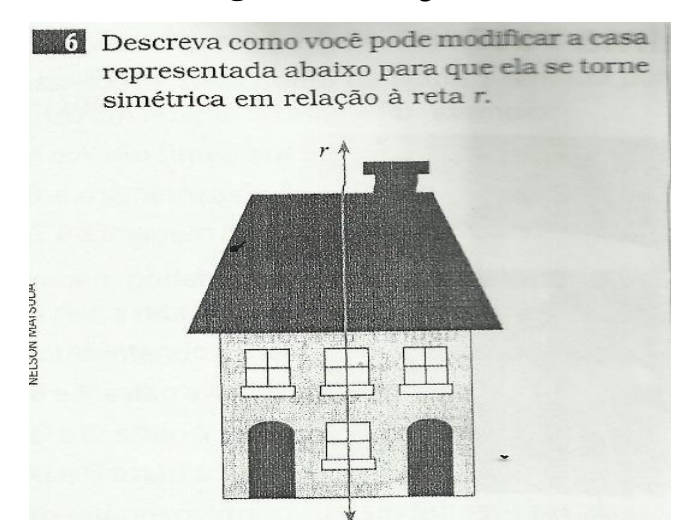

Fonte: Bianchini(2006, p.162) 
Diferentemente dos livros anteriores, este, L3, livro didático do $8^{\circ}$ ano apresenta atividades que se classificam até o nível da dedução. Sendo assim, notamos que os níveis da visualização análise dedução informal estão inclusos, pelo fato destes serem alcançados mediante conhecimentos hierárquicos. A seguir, apresentaremos a figura 03.

Figura 03: Exercícios propostos

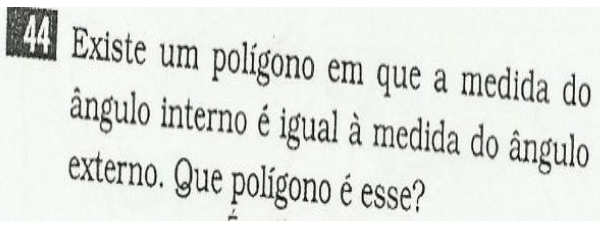

Fonte: Bianchini (2006, p.107)

No L4, diante nossa análise, verificamos que este livro apresenta atividades que se enquadram no nível da dedução informal, mas que verificamos que para resolve-los é necessários os conhecimentos geométricos dos níveis anteriores, como, por exemplo, visualização e análise.

Figura 04: Exercícios propostos sobre polígonos

30 o professor de Matemática de uma escola promoveu um campeonato de pipas entre os alunos. Para isso, passou a seguinte especificação: a pipa déverá ter a forma de um hexágono regular com lados medindo $20 \mathrm{~cm}$. Calcule, em seu caderno, a medida do apótema e a área da pipa.

Fonte: Bianchini (2006, p.225)

Portanto, considerando que a análise foi realizada com os cinco níveis de compreensão do modelo de Van Hiele, afirmamos que o nível rigor não esteve presente, acreditamos que seja pelo fato deste nível conceber que os alunos já são capazes de comparar sistemas baseados em diferentes axiomas, além disto, diante das análises realizadas, verificamos que não houve atividades que estavam relacionadas a este nível. Além disto, consideramos que este não estejam presentes pelo fato do nível da escolaridade este não venha a ser enquadrar, visto que neste nível os alunos “já são capazes de comparar sistemas baseados em diferentes axiomas" (ALVES; SAMPAIO, 2010, p.70). 
Diante das análises realizadas sobre as atividades dos polígonos nos livros didáticos, a seguir, apresentaremos, mediante a análise realizada um quadro resumindo os níveis de compreensão de Van Hiele, adotados nas atividades apresentadas nos livros didáticos.

Quadro 3: Níveis de Compreensão adotados a partir das análises realizadas das atividades sobre polígonos nos livros didáticos.

\begin{tabular}{|c|c|c|}
\hline $\begin{array}{c}\text { Livro } \\
\text { didático }\end{array}$ & $\begin{array}{l}\text { Níveis de compreensão } \\
\text { de Van Hiele }\end{array}$ & Características da Atividades \\
\hline & Visualização & $\begin{array}{l}\text { Reconhecimento de linhas poligonais e } \\
\text { Reconhecimento de polígonos }\end{array}$ \\
\hline L1 & Análise & $\begin{array}{l}\text { Identificação de polígonos convexos e não convexos; } \\
\text { Identificação de linhas poligonais abertas e fechadas e } \\
\text { Identificação de propriedades dos polígonos. }\end{array}$ \\
\hline \multirow[t]{2}{*}{$\mathbf{L 2}$} & Visualização & Atividade envolvendo o reconhecimento de figura geométrica. \\
\hline & Análise & Atividade sobre propriedades dos polígonos \\
\hline \multirow[t]{3}{*}{$\mathbf{L 3}$} & $\begin{array}{l}\text { Visualização e } \\
\text { Análise }\end{array}$ & $\begin{array}{l}\text { As atividades, neste livro didático, rementem- se ao nível } 4 \\
\text { porém para resolve- las os alunos tem que conceber } \\
\text { conhecimentos prévios dos primeiros níveis, como, por } \\
\text { exemplo o reconhecimento de figura geométrica } \\
\text { (Visualização), identificação das propriedades da figura } \\
\text { (análise), para realizar as "Provas", isto é as demonstrações } \\
\text { que são apresentadas nas atividades }\end{array}$ \\
\hline & Dedução Informal & $\begin{array}{l}\text { Faz inclusão de classes, assim como acompanhar provas } \\
\text { formais que posteriormente não conseguem realiza outras. }\end{array}$ \\
\hline & Dedução & $\begin{array}{l}\text { Realização de "Provas", ou seja demonstrações de } \\
\text { congruência de ângulos, como também lados. }\end{array}$ \\
\hline \multirow[t]{2}{*}{$\mathbf{L 4}$} & Visualização e Análise & $\begin{array}{c}\text { Para resolução das atividades, mesmo sabendo que elas } \\
\text { pertencem ao nível da Dedução Informal é perceptível, que } \\
\text { para resolve- las os alunos precisam compreender os níveis da } \\
\text { visualização e análise. }\end{array}$ \\
\hline & Dedução Informal. & $\begin{array}{l}\text { Realização de algumas provas formais, mas os alunos ainda } \\
\text { não são capazes de construir outra. }\end{array}$ \\
\hline
\end{tabular}

Fonte: Elaborado pelo autor (2014)

Como podemos perceber, o nível rigor não esteve presente durante as análises realizadas das atividades, pois acreditamos que seja pelo fato deste nível conceber que os alunos já são capazes de comparar sistemas baseados em diferentes axiomas, além disto, 
diante das análises realizadas, verificamos que não houve apresentação de atividades que estavam relacionadas a este nível.

\section{Considerações finais}

Como retratado no início desta pesquisa, elencamos que esta constituiu-se a partir de uma abordagem de cunho qualitativa, mediante estudo de análise documental, pois, prezamos em analisar uma coleção de livros didáticos dos anos finais do ensino fundamental que foram os mais utilizados no município de São Vicente Férrer/ PE.

Com base em nossa análise, podemos perceber que os livros didáticos de forma geral abordam durante todo o Ensino Fundamental os conteúdos dos polígonos e além disto as atividades analisadas seguem em grande parte nosso referencial teórico, Van Hiele.

Com apoio dos níveis de compreensão do Modelo de Van Hiele, salientamos que ampliamos nossos conhecimentos sobre a importância de se trabalhar no ensino fundamental o conteúdo de geometria, em especial os conceitos geométricos. Embora, de acordo com nossa fundamentação teórica percebemos que os ensinos da geometria muitas vezes são relegados para o segundo plano, e por consequência disto este conhecimento não é lecionado.

Diante da análise realizada das atividades dos livros didáticos, foi verificado que no L1, encontramos os níveis de Visualização e Análise; no L2, os níveis de Visualização e Análise, no L3, os níveis visualização, análise, dedução informal e dedução; e no L4 os níveis da visualização, análise e dedução informal, e, além disto que o nível denominado de rigor não foi apresentada em nenhum dos livros, percebemos que seja pelo fato de sua complexidade quanto ao nível de estudo.

Investigar a proposta do ensino de polígonos em uma coleção de livro didático foi de grande relevância para nós, pois em muitas instituições o livro didático, por sua vez é a sua única fonte de consulta disponível para o processo de ensino- aprendizagem, sendo assim os conteúdos ao serem apresentados tem que ser de forma clara para entendimento do aluno.

Com base em nossa análise, verificamos que os nossos objetivos foram alcançados, assim como a problema de pesquisa, visto que usamos como aporte teórico os níveis de compreensão do Modelo de Van Hiele.

Ainda, de acordo com a análise de dados obtidos da pesquisa, vale salienta que este instrumento (os livros didáticos) é de grande relevância para futuros trabalhos, principalmente para profissionais da educação que já os utilizam, como recurso didático. Entretanto, podemos 
elencar ainda que, mediantes futuras pesquisa esta fosse conduzida em um estudo de caso, englobando os livros didáticos, professores e alunos. Pois, seria possível perceber a relação sobre a proposta de ensino adotados nos livros, a aprendizagem dos alunos e por fim como o professor conduz este ensino, e com isto poderia utilizar teorias como, por exemplo, o modelo de Van Hiele como instrumento didático-metodológico e entre outros.

Logo, espera-se que este trabalho centrado na investigação das relações estabelecidas entre a proposta de ensino de polígonos e o modelo de Van Hiele, seja, de certa forma, uma ferramenta de uso de novas pesquisas.

\section{Referências}

ALVES, George de Souza; SAMPAIO, Fábio Ferreira. O modelo de desenvolvimento do pensamento geométrico de Van Hiele e possíveis contribuições da geometria dinâmica. Revista de Sistemas de Informação da FSMA, n.5 (2010), pp. 69 - 76.

ARAÚJO, Ivanildo do Basílio de. Uma abordagem para a prova com construções geométricas e cabri-geométre. Dissertação (Mestrado em Ensino de Matemática) Pontifícia Universidade Católica de São Paulo- PUC. São Paulo, 2007.

BIANCHINI, Edwaldo. Matemática. 6 edições. São Paulo: Moderna, 2006.

BRASIL, Secretaria de Educação Fundamental. Parâmetros Curriculares Nacionais: matemática/ Secretaria de Educação Fundamental- Brasília: Mec/ sef,1998.

CHIZZOTTI, Antônio. Pesquisa qualitativa em ciências humanas e sociais: evolução e desafios. Revista Portuguesa de Educação, año/ vol. 16, número 002, Universidade do Minho Braga, Portugal, 2003, pp. 221- 236.

GIL, Antônio Carlos. Métodos e técnicas de pesquisa social. - 6. ed. - São Paulo: Atlas, 2008. GOLDENBERG, Mirian. A arte de pesquisar: como fazer pesquisa qualitativa em ciências sociais. Rio de janeiro: Record, 2007.

GUERATO, Elisabete Teresinha. Dificuldades e possibilidades no ensino da geometria na EJA. Centro Federal de Educação Tecnológico de São Paulo. São Paulo, Março de 2008.

GUIMARÃES, Rosângela Resende. Monografia um estudo do pensamento geométrico de professores das séries iniciais do ensino fundamental segundo o modelo de Van Hiele. Universidade Federal de Minas Gerais, departamento e matemática, especialização para professores. Belo Horizonte, 2006.

INOUE, Rosa Kazuko Miyasaki. O processo de formação do conceito de quadriláteros, envolvendo alunos de uma $6^{a}$ série do ensino fundamental. Universidade do vale do itajai, curso de pós-graduação scricto sensu. Itajaí, 2004.

LORENZATTO A. Por que não ensinar geometria? A educação Matemática em revista - ano III- n $^{\circ}$ 4- publicação da sociedade brasileira de educação matemática, 1995. 
PERES, G. Pressupostos e reflexões teóricas e metodológicas da pesquisa participante no ensino da Geometria para camadas populares ( $1^{\circ}$ e $2^{\mathbf{o}}$ graus). Tese (doutorado). Unicamp, Campinas, 1991.

RODRIGUES, Alessandra Coelho, Universidade Católica de Brasília; Curso de Matemática; Orientador: Vilmondes Rocha. O modelo de Van Hiele de desenvolvimento do pensamento geométrico. em: http://www.ucb.br/sites/100/103/TCC/22007/AlessandraCoelhoRodrigues.pdf. Acesso em:17/ 04/ $\underline{2014}$

SILVA, João Everton Cezário da. O ensino de polígonos e a teoria de Van Hiele. Universidade Federal da Paraíba; Universidade aberta do Brasil; Centro de Ciências Exatas e da Natureza; Departamento de Matemática; Curso de Licenciatura em Matemática a Distância. João Pessoa - PB, 2011.

VIEIRA, Carmem Rosilene. Reiventando a geometria no ensino médio [manuscrito]: uma abordagem envolvendo materiais concretos, softwares de geometria dinâmica e a teoria Van Hiele. 2010. XII, 155f: il color.

Recebido em 25/03/2017 - Aceito em 06/11/2017 